\title{
SYNCHROTRON X-RAY DIFFRACTION IN ATYPICAL MACULAR DYSTROPHY
}

\author{
ANDREW J. QUANTOCK ${ }^{1}$, KEITH M. MEEK ${ }^{2}$, EUGENE J.-M. A. THONAR ${ }^{3}$ and KERRY K. ASSIL ${ }^{1}$ \\ St Louis, Oxford and Chicago
}

\begin{abstract}
SUMMARY
This report documents the first case of X-ray diffraction techniques aiding the diagnosis of a corneal dystrophy with a clinically ambiguous presentation. Post-operatively, a high-angle synchrotron $\mathrm{X}$-ray diffraction pattern was obtained from an in vitro portion of a pathological cornea. This pattern displayed two $\mathrm{X}$-ray reflections which we recently demonstrated to be unique to the high-angle X-ray diffraction patterns of both type I and type II macular dystrophy corneas; on the basis of this evidence we were able to offer a post-operative diagnosis of macular corneal dystrophy. An electron microscopical evaluation of the cornea revealed stromal lacunae at all levels and an extensive layer of vacuoles, predominantly between Bowman's layer and the anterior stroma. These vacuoles were often associated with large proteoglycan filaments, as identified by Cuprolinic blue staining. Abnormally large collagen fibrils were documented, for the first time, in a macular dystrophy cornea; they existed in localised regions, frequently adjacent to the vacuoles and abnormal proteoglycans, and could well have implications for corneal transparency. We propose that the dystrophy is an atypical variant of macular corneal dystrophy which is encompassed by the heterogeneous nature of the condition.
\end{abstract}

A 75-year-old adopted man, who 5 years previously was reported visually normal by his referring physician, underwent penetrating keratoplasty of the right eye for an unusual bilateral corneal disorder. On the basis of the clinical findings the disorder appeared dystrophic, and a tentative diagnosis of granular or macular corneal dystrophy was entertained.

Macular corneal dystrophy is a rare, autosomal recessive disorder, classically presenting within the first decade

From: ${ }^{1}$ Anheuser-Busch Eye Institute, Saint Louis University School of Medicine, St Louis,.. Missouri, USA; ${ }^{2}$ Biophysics Group, Open University, Oxford Research Unit, Oxford, UK; ${ }^{3}$ Departments of Biochemistry and Internal Medicine, Rush Medical Center, Chicago, Illinois, USA.

Correspondence to: Andrew J. Quantock, PhD, Anheuser-Busch Eye Institute, Department of Ophthalmology, Saint Louis University, 1755 S. Grand Blvd., St Louis, MO 63104, USA. of life and becoming symptomatically advanced by the third decade. The corneal stroma becomes progressively clouded with central superficial deposits which eventually extend posteriorly and towards the limbus. ${ }^{1}$ Central corneal thinning is evident, ${ }^{2.3}$ associated with the compaction of normal-diameter stromal collagen fibrils. ${ }^{4}$

Until about 5 years ago, the primary defect in macular corneal dystrophy was assumed to occur in the synthetic pathway of the keratan sulphate proteoglycans, ${ }^{5-7}$ and was thought always to involve an incomplete glycosaminoglycan sulphation process. ${ }^{7}$ However, in 1988 immunochemical studies evaluating the keratan sulphate defect in a population of macular dystrophy patients revealed a heterogeneity. ${ }^{8,9}$ Briefly, the cornea and serum of some patients (designated as type I) contain no sulphated keratan sulphate, as identified by a monoclonal antibody in an ELISA, ${ }^{10.11}$ whereas other patients (designated as type II) demonstrate measurable, though often reduced, keratan sulphate levels in either or both of these tissues. ${ }^{8,9}$ Additional reports of individual cases which defy this classification system ${ }^{8}$ emphasise the heterogeneous nature of macular corneal dystrophy. Macular dystrophy corneas synthesise chondroitin/dermatan sulphate proteoglycans that are larger than normal and oversulphated, ${ }^{7}$ and histological studies employing the proteoglycan-specific stain Cuprolinic blue have revealed that these proteoglycans accumulate and aggregate in both type I and type II macular dystrophy corneas. ${ }^{4,12}$

Corneal stroma produces an X-ray diffraction pattern, the high-angle component of which relates to the arrangement of atoms and groups of atoms into molecules, and of collagen molecules into fibrils. Macular dystrophy corneas produce a unique high-angle synchrotron X-ray diffraction pattern, ${ }^{13}$ possibly due to the abnormal ultrastructure of the stromal proteoglycans. This phenomenon was recently put forward as an alternative to the histological approach of post-operatively confirming a diagnosis of macular corneal dystrophy. ${ }^{13}$

Given the patient's extremely unusual clinical presentation, we performed transmission electron microscopy in 


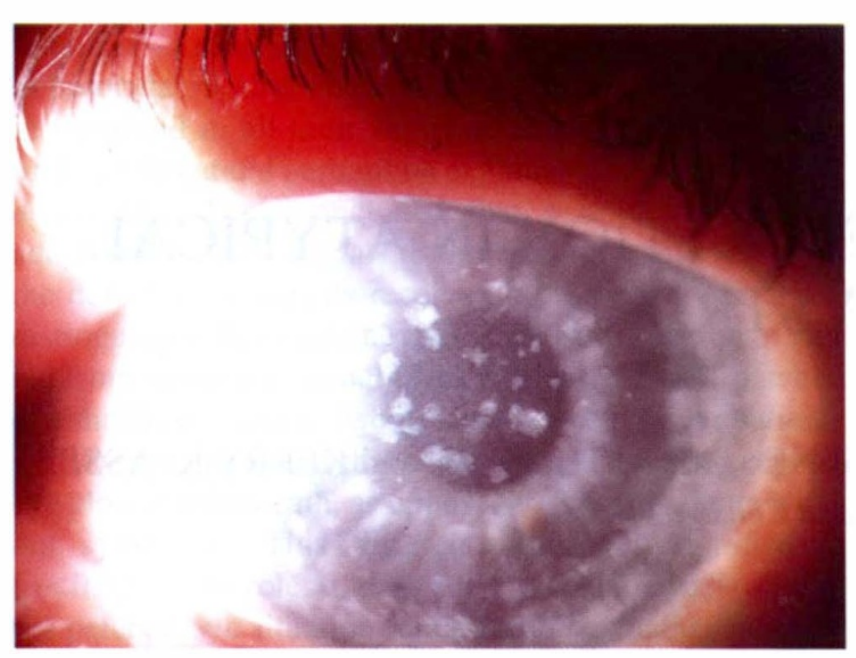

Fig. 1. The clinical presentation of the cornea just prior to penetrating keratoplasty. Small, white opacities with somewhat distinct margins are present in the superficial central stroma with some intervening stromal clouding.

conjunction with proteoglycan-specific staining to discern the ultrastructural abnormalities of his cornea. Blood serum and corneal tissue were analysed for epitopes of sulphated keratan sulphate. We also obtained high-angle synchrotron X-ray diffraction patterns from the corneal stroma. This report represents the first incidence of X-ray diffraction data aiding the diagnosis of a corneal pathology with a clinically ambiguous presentation.

\section{MATERIALS AND METHODS}

Immediately upon resection of the $7.5 \mathrm{~mm}$ central corneal button, three $2 \mathrm{~mm}$ diameter punch biopsies were taken from the central affected area and processed for transmission electron microscopy as described below. A corneal portion and a serum sample were frozen prior to the keratan sulphate analysis. The remainder of the button, a portion of which was earmarked for use in the X-ray diffraction study, was fixed overnight at $4^{\circ} \mathrm{C}$ in $4 \%$ paraformaldehyde in $0.1 \mathrm{M}$ cacodylate buffer $(\mathrm{pH}$ 7.4) before being stored in the buffer without the fixative.

\section{Keratan Sulphate Analysis}

The keratan sulphate content of a sample of cornea and serum was analysed by a previously described ELISA which employs a monoclonal antibody specific for a very highly sulphated epitope on the keratan sulphate chain; several normal adult human corneas were identically treated. ${ }^{11}$ The results are expressed as equivalents of an international standard of keratan sulphate purified from human costal cartilage.

\section{$X$-ray Diffraction}

$X$-ray diffraction patterns were recorded on high-speed photographic film, using the high-angle $(\approx 12 \mathrm{~cm})$ camera and radiation of wavelength $1.488 \AA$, at the SERC synchrotron in Daresbury, UK. The diffraction system was calibrated from the $3.04 \AA$ lattice reflection in powder diffraction patterns of calcite, and analysis of the X-ray pat- terns was as previously described. ${ }^{13}$ The confidence limits are expressed as the precision with which the reflection could be measured, converted to real space. As the data are converted from reciprocal space, the wider reflections produce Bragg spacings which are associated with a lower experimental error.

\section{Transmission Electron Microscopy}

The three corneal punches were fixed, stained en bloc for proteoglycans with Cuprolinic blue in a 'critical electrolyte concentration' mode $\left(0.1 \mathrm{M} \mathrm{MgCl}_{2}\right)$ and processed for electron microscopy as previously described..$^{14}$ The grids were examined on a Jeol $100 \mathrm{~S}$ operating at $80 \mathrm{kV}$, calibrated with a 2160 lines/mm grating replica.

\section{RESULTS}

\section{Clinical Presentation}

Slit lamp biomicroscopic examinations revealed multiple, dense, white opacities with somewhat distinct margins in the superficial central stroma with some intervening stromal clouding (Fig. 1). Mid-peripheral stroma was relatively unaffected. In the peripheral cornea the superficial stroma was clear, and discrete, smooth pre-Descemet's lesions were evident. Routine pathology indicated an acquired phenotypical mucopolysaccharidosis.

\section{Keratan Sulphate Analysis}

Serum keratan sulphate was quantified at $390 \mathrm{ng} / \mathrm{ml}$

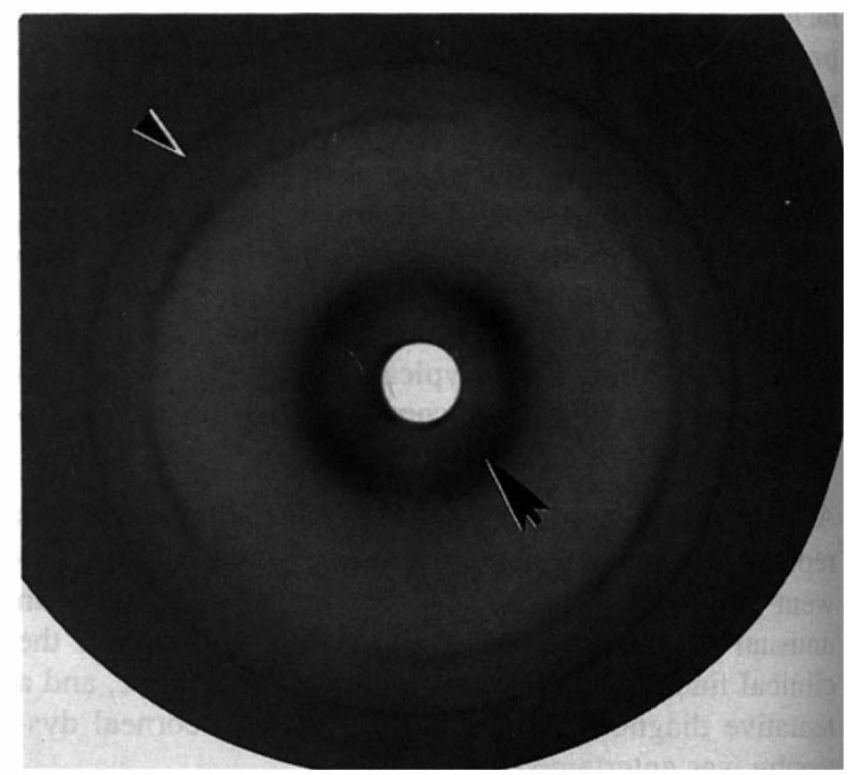

Fig. 2. A high-angle synchrotron X-ray diffraction pattern from a portion of the cornea. The diffuse inner reflection (arrow) arises from the lateral separation of collagen molecules (in this case $15.1 \pm 0.4 \AA$ ). Two sharp reflections which are unique to macular dystrophy corneas are observed (outer reflection; arrowhead), although the inner of the two is extremely faint; these reflections correspond to Bragg spacings of $4.56 \pm 0.05 \mathrm{~A}$ (outer reflection) and $9.4 \pm 0.3 \AA$ (inner reflection). The diffuse reflection just inside the outer 'extra' reflection is due to the mylar window of the experimental cell in which the cornea is placed during the exposure. 


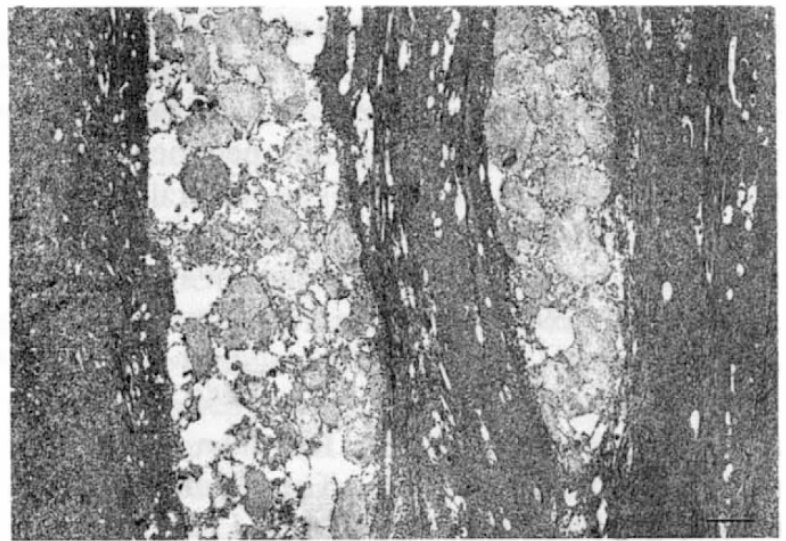

Fig. 3. A low-magnification $(\times 7800)$ micrograph of the abnormal vacuolated layer interspersed with anterior stroma. Bowman's layer is to the far left of the image, and in this region there is some stroma anterior to the vacuolated layer. Electronlucent lacunae are present within the vacuolated layer and the stroma. Large, darkly stained proteoglycan filaments are associated with the margins of the vacuoles. Scale bar represents $1.4 \mu \mathrm{m}$.

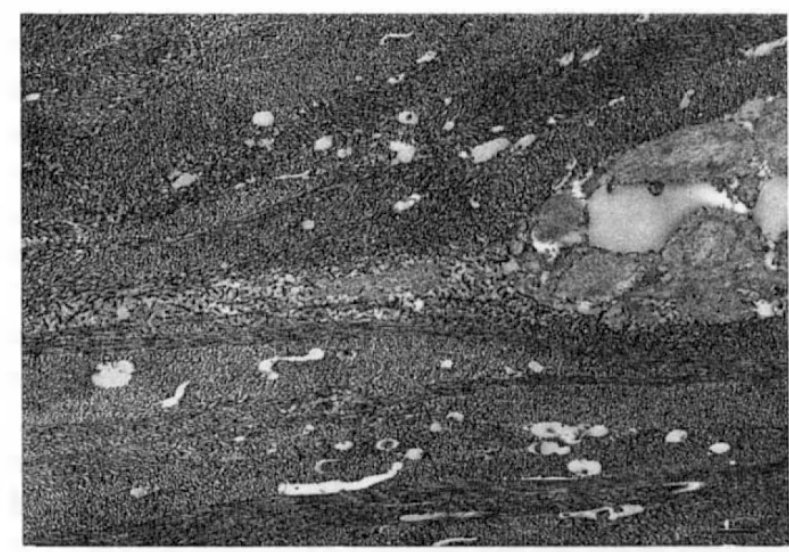

Fig. 5. A region of mid-depth stroma which displays numerous electron-lucent lacunae, both inter-lamellar and intralamellar. Stromal proteoglycans appear fairly normal. A deposit of vacuoles lies between two lamellae, and the abnormally large proteoglycan filaments extend into the adjacent stromal matrix $(\times 17$ 300). Scale bar represents $500 \mathrm{~nm}$.

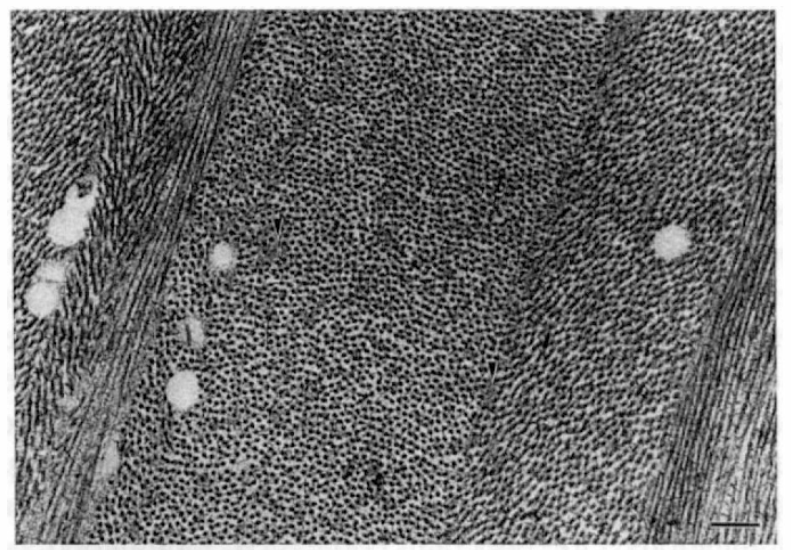

Fig. 7. A micrograph $(\times 21000)$ of the mid-stroma displaying normal-diameter collagen fibrils in a lamellar arrangement. Electron-lucent lacunae are present in the matrix. Similar-sized, diffusely stained stromal inclusions are also evident (arrowheads). Scale bar represents $250 \mathrm{~nm}$.

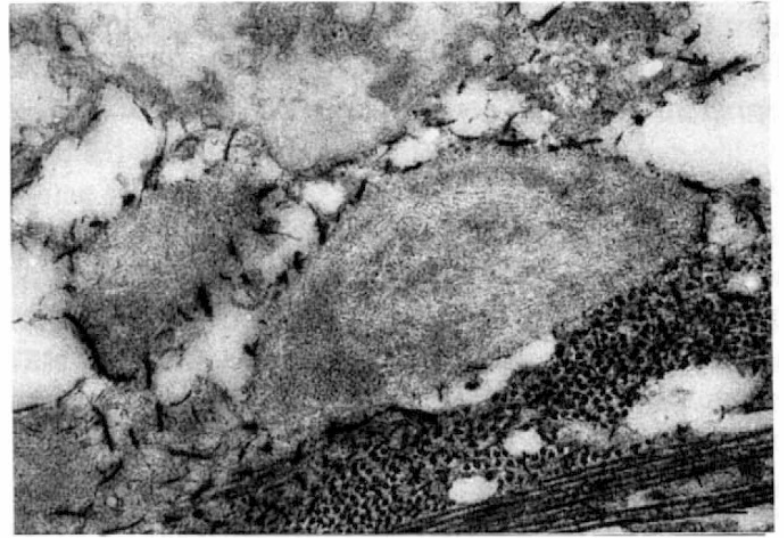

Fig. 4. A high-magnification (×43 200) micrograph of vacuoles adjacent to the anterior stroma. Stromal proteoglycans appear normal. The vacuoles are seen to have a vaguely discernible structure and large proteoglycan filaments are associated with the periphery of the vacuoles. Scale bar represents $200 \mathrm{~nm}$.

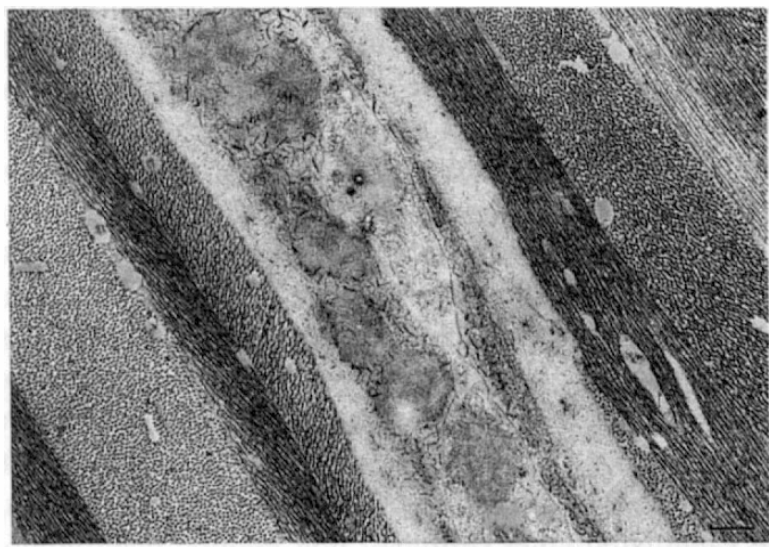

Fig. 6. A micrograph $(\times 40000)$ of vacuoles in close approximation to a mid-stromal keratocyte. Abnormally large proteoglycan filaments are associated with the margins of the majority of the vacuoles in this micrograph; these vacuoles do not seem to be bound by a membrane. A vacuole which is enclosed within a membrane does not have any proteoglycans associated with it. Scale bar represents $250 \mathrm{~nm}$.

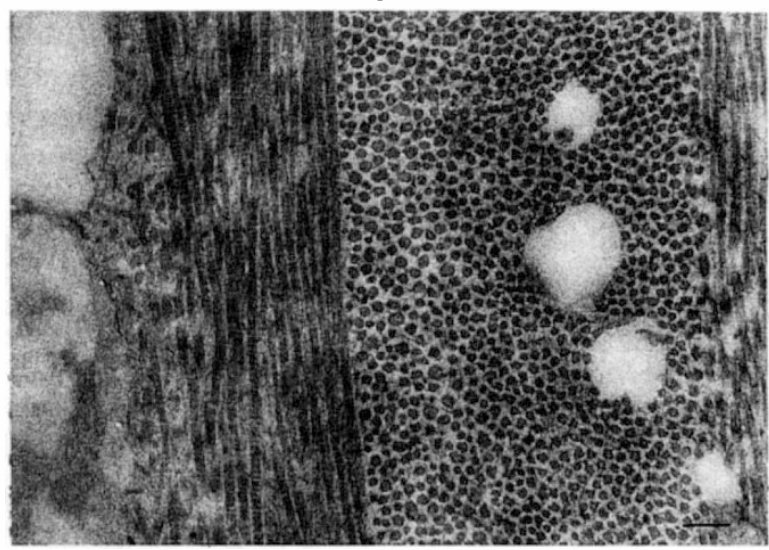

Fig. 8. A region of anterior stroma just posterior to a vacuolated layer ( $\times 53$ 300). The vacuolated layer lies to the left of the image. Matrix proteoglycans appear fairly normal and electron-lucent lacunae are in evidence. The collagen fibrils, in cross-section and long-section, demonstrate an abnormally large variation in their diameters. The lamella viewed in cross-section displays the most variability in its collagen sizes towards the left, i.e. in the region most closely approximated to the vacuolated layer and the abnormal proteoglycan filaments. Scale bar represents $200 \mathrm{~nm}$. 
(normal, $265 \mathrm{ng} / \mathrm{ml}^{11}$ ). Keratan sulphate constituted 35\% of the corneal proteoglycans compared with $40-60 \%$ in normal adult human cornea.

\section{Synchrotron X-ray Diffraction}

Analysis of the high-angle X-ray diffraction pattern from the cornea (Fig. 2) demonstrates that the mean distance between the collagen molecules which constitute the stromal fibrils is $15.1 \pm 0.4 \AA$. More importantly, two 'extra' reflections are observed which correspond to Bragg spacings of $4.56 \pm 0.05 \AA$ and $9.4 \pm 0.3 \AA$.

\section{Transmission Electron Microscopy}

A collagen-free layer is present in the central anterior cornea, predominantly between Bowman's layer and the anterior stroma, although occasionally it extends beneath the anterior stroma (Fig. 3). This layer consists of numerous circular and oval vacuoles (Fig. 3) which are filled with fibrillogranular material with a vaguely discernible ultrastructure (Fig. 4). The dimensions of these vacuoles are approximately 1-4 $\mu \mathrm{m}$. Electron-lucent areas of similar proportions are interspersed with the vacuoles (Figs. 3-5). The abnormal fibrillogranular layer, which is up to $22 \mu \mathrm{m}$ thick, is observed in all of the samples taken from the central cornea. Abnormally large, Cuprolinicblue-stained proteoglycan filaments $(20-40 \mathrm{~nm}$ thick and $50-400 \mathrm{~nm}$ long) are present within this layer, predominantly associated with the outer edge of the individual vacuoles (Figs. 3-5); these proteoglycan filaments often extend into the stromal matrix adjacent to the margins of the vacuolated layer (Fig. 5).

Numerous, discrete lacunae occur at all levels of the corneal stroma and, occasionally, an undefined diffuse substance is observed within this type of lacuna (Fig. 6). Similar, smaller structures are present in Bowman's layer. Occasionally, mid-stromal keratocytes are closely approximated to fibrillogranular vacuoles (Fig. 7). The extracellular vacuoles, which lie in close proximity to the cell, but do not seem to be bound by a membrane, are frequently associated with large proteoglycan filaments. Vacuoles which appear to be in the cytoplasm do not display the proteoglycan association (Fig. 7).

The general size and distribution of the stromal collagen and proteoglycans throughout most of the stroma appears normal (Figs. 5-7). However, localised regions of the stroma occasionally display a large variation in the diameter (approximately 25-60 $\mathrm{nm}$ ) of their collagen fibrils (Fig. 8). In the electron microscope normal human corneal collagen fibrils have a mean diameter of $26 \mathrm{~nm}$ $(\mathrm{SD} \pm 2.4 \mathrm{~nm}){ }^{15}$ The abnormally large collagen fibrils seem always to occur adjacent to abnormal vacuolated regions containing the large proteoglycan filaments.

\section{DISCUSSION}

The patient's history, and the clinical appearance of the cornea, had some characteristics of macular corneal dystrophy, although they were atypical of the condition. The patient's age at presentation is, to our knowledge, older than any previously reported case of macular corneal dystrophy ${ }^{8-11,16}$ which generally presents earliest of the classic stromal dystrophies. The central opacities are confined to the superficial stroma while the peripheral opacities exist in the deep, pre-Descemet's stroma. Furthermore, it is unusual for the opacities in macular dystrophy corneas to have such distinct margins. The patient's serum keratan sulphate levels $(390 \mathrm{ng} / \mathrm{ml})$ were well above those for type I macular corneal dystrophy $(<3 \mathrm{ng} / \mathrm{ml})$, and normally sulphated keratan sulphate constituted $35 \%$ of the corneal proteoglycans compared with less than $1 \%$ in type I macular dystrophy corneas. These immunochemical findings preclude diagnosis of type I macular corneal dystrophy but allow for type II macular corneal dystrophy.

We previously noted that a striking feature of $\mathrm{X}$-ray diffraction patterns obtained from macular dystrophy corneas is the presence of either one or two sharp 'extra' $\mathrm{X}$-ray reflections. ${ }^{13}$ We also discovered these 'extra' reflections to be independent of the macular corneal dystrophy subtype and the particular pedigree involved. The occurrence and position of the outer reflection from both type I and type II macular dystrophy corneas is remarkably consistent: it is present without exception and arises from a regularly repeating structure with a periodicity of $4.61 \AA$ ( $S D 0.03 \AA$ ). The position of the inner reflection is no less consistent, arising as it does from a $9.62 \AA$ (SD $0.04 \AA$ ) periodic structure, though it is only recorded at lower corneal hydrations. In this study the analysis of the $\mathrm{X}$-ray diffraction pattern revealed the average separation of the stromal collagen molecules to be approximately $15 \AA$. This intermolecular spacing implies that, at the time the X-ray diffraction pattern (Fig. 2) was obtained, the hydration of the cornea (defined as the ratio of the weight of corneal water to dry corneal tissue) was below 0.5 , a level at which a macular dystrophy cornea would be expected to display the inner $(9.62 \AA)$ ' 'extra' reflection. ${ }^{13}$ 'Extra' reflections from the present study are observed at $4.57 \pm 0.05 \AA$ and $9.4 \pm 0.3 \AA$. These reflections are, within the experimental resolution of the $\mathrm{X}$-ray diffraction system, sufficiently similar to the characteristic macular dystrophy reflections to lead us to infer that they arise from the same, or similar, phenomenon. We suspect this phenomenon to be the abnormal sulphation of the glycosaminoglycan chains, but this remains to be proven. The exact origin of the reflections notwithstanding, they remain unique to macular dystrophy corneas. 'Extra' reflections were present on every X-ray diffraction pattern from six distinct macular dystrophy corneas,${ }^{13}$ and were not detected on any patterns from 28 normal human corneas. Moreover, patterns from a Reis-Bückler's cornea, a granular dystrophy cornea (D. W. Leonard, personal communication), 21 keratoconus corneas (N. J. Fullwood, personal communication), 7 oedematous corneas ${ }^{17}$ and a Scheie's syndrome cornea ${ }^{15}$ were devoid of the characteristic 'extra' reflections. Thus, in this case we are able to offer the first diagnosis of macular corneal dystrophy based on X-ray diffraction evidence. The present X-ray diffraction study further demonstrates that chemical fix- 
ation of the tissue does not appreciably modify the 'extra' reflections. Currently, detection of the outer $(4.61 \AA)$ 'extra' reflection has only been performed on a highintensity synchrotron radiation source (SRS, Daresbury, UK). There is, however, no reason why a laboratory-based $\mathrm{X}$-ray set could not detect the reflection although the predicted exposure time is in the order of many hours. Nevertheless, with the advancement of X-ray technology it should be possible to detect the outer 'extra' reflection with more convenient exposure times.

The anterior stromal fibrillogranular-filled vacuoles are, as revealed by electron microscopy, ultrastructurally similar to previously documented deposits in macular dystrophy corneas. ${ }^{16,18}$ However, in this case, the abnormal region is much more extensive (up to approximately $4 \%$ of the total corneal thickness) since the vacuoles, which are of a similar size to those previously reported, are more numerous. The electron-lucent areas which coexist with the fibrillogranular-filled vacuoles are of similar dimensions to the vacuoles themselves and may represent vacuoles whose fibrillogranular material was extracted during the tissue processing. That fibrillogranular material is present in mid-stromal keratocytes suggests that it forms intracellularly, and is somehow extruded into the extracellular matrix by the cell, or remains as cellular debris when the cell dies. It is not entirely clear why large proteoglycan filaments are associated with the margins of the extracellular anterior and mid-stromal vacuoles but not the intracellular ones.

The large extent of the anterior vacuolated region is presumably due to the advanced stage of the disease in this patient, who was 75 years old at the time of surgery. We can postulate that accumulation of the vacuoles began early in life and advanced gradually, in which case the late onset of the symptoms implies that the contribution of the abnormal vacuolated region to corneal opacification is limited. However, reasoning suggests that the extensive vacuolated region contributes to corneal opacification, and thus argues in favour of this particular case representing the late onset and rapid development of a form of macular corneal dystrophy.

A new finding of this study is that the abnormally large proteoglycan filaments associate predominantly with the periphery of the vacuoles in the anterior cornea. These proteoglycan filaments, as detected by Cuprolinic blue staining, are similar to those identified in the stroma of both type I and type II macular dystrophy patients. ${ }^{4,12} \mathrm{We}$ previously identified these abnormally large filaments as chondroitin/dermatan sulphate, ${ }^{12}$ and surmised that they represented proteoglycan aggregates rather than individual molecules. The present case further specifies that the large proteoglycan filaments are primarily confined to the abnormal superficial layer, and, with the exception of those proteoglycan filaments associated with the vacuoles along the margin of the mid-stromal keratocytes, are not diffusely distributed throughout the extracellular stromal matrix.

Numerous, well-defined, convoluted lacunae are pres- ent throughout the entire stromal matrix, both within and between lamellae. Similar lacunae have been observed in previous work $^{4,12}$ but, unlike the present case, these often contained large proteoglycan filaments. In general, the lacunae in this case measure up to approximately $1 \mu \mathrm{m}$ across. The transparency theory of Benedek ${ }^{19}$ proposes that when the size of a lacuna approaches half the wavelength of light (approximately $250 \mathrm{~nm}$ ) it will scatter light. Evidently, in this case some of the stromal lacunae should theoretically contribute to corneal cloudiness and the patient's visual disturbance.

Evidence exists which implicates corneal proteoglycans in the modulation of both the regular distribution ${ }^{20,21}$ and the uniform diameter ${ }^{22.23}$ of corneal collagen fibrils. However, some work proposes that the effect of stromal proteoglycans on the regulation of the fibril diameter is limited, and that the proportion of type V collagen is the dominant factor. ${ }^{20,24.25}$ Previous ultrastructural studies of macular dystrophy corneas ${ }^{4,12}$ have revealed normal-diameter stromal collagen fibrils. This report is the first to document localised regions of heterogeneous, abnormally large diameter collagen fibrils. The range of fibril diameters is similar to the range recently observed in the stroma of a patient with Scheie's syndrome. ${ }^{15}$ The present study shows that the regions of localised large collagen often occur adjacent to regions containing large proteoglycan filaments. Unless some undetermined factors, such as low levels of type $\mathrm{V}$ collagen, are involved the implication from this work, as from the Scheie's syndrome study, ${ }^{15}$ is of an association between abnormal, in vivo, proteoglycan content and localised regions of large-diameter collagen fibrils. These localised regions of largediameter collagen fibrils could well have implications for corneal transparency.

In this study, X-ray diffraction techniques were employed, for the first time, in the post-operative diagnosis of a clinically ambiguous pathological condition. We conclude that the condition described in this report is an atypical dystrophy which is encompassed by the heterogeneous nature of the diseases classed as macular corneal dystrophies.

The authors acknowledge the technical expertise of Mary Ellen Lenz. This work was financially supported by MRC grant G8818113CB (KMM), NIH grants AG04736 and AR39239 (EJMAT) and an unrestricted grant to Anheuser-Busch Eye Institute, Saint Louis University from Research to Prevent Blindness, Inc. The Open University's Biophysics Group acknowledges the financial assistance of the T.F.C. Frost Charitable Trust.

Key words: Collagen, Macular corneal dystrophy, Proteoglycans.

\section{REFERENCES}

1. Klintworth GK. Research into the pathogenesis of macular corneal dystrophy. Trans Ophthalmol Soc UK 1980;100: 186-94.

2. Ehlers N, Thorkild B. Central thickness in corneal disorders. Acta Ophthalmol (Copenh) 1978;56:412-6.

3. Donnenfeld ED, Cohen EJ, Ingraham HJ, Poleski SA, Gold- 
smith E, Laibson PR. Corneal thinning in macular corneal dystrophy. Am J Ophthalmol 1986;101:112-3.

4. Quantock AJ, Meek KM, Ridgway AEA, Bron AJ, Thonar EJ-MA. Macular corneal dystrophy: reduction in both corneal thickness and collagen interfibrillar spacing. Curr Eye Res 1990;9:393-8.

5. Klintworth GK, Smith CF. Macular corneal dystrophy: studies of sulphated glycosaminoglycans in corneal explant and confluent stromal cell cultures. Am J Pathol 1977;89: $167-82$.

6. Hassell JR, Newsome DA, Krachmer JH, Rodrigues M. Macular corneal dystrophy: failure to synthesize a mature keratan sulphate proteoglycan. Proc Natl Acad Sci USA 1980;77:3705-9.

7. Nakazawa K, Hassell JR, Hascall VC, Lohmander S, Newsome DA, Krachmer J. Defective processing of keratan sulphate in macular corneal dystrophy. J Biol Chem 1984;259: 13751-7.

8. Edward DP, Yue BYTJ, Sugar J, et al. Heterogeneity in macular corneal dystrophy. Arch Ophthalmol 1988;106: 1579-83.

9. Yang CJ, SundarRaj N, Thonar EJ-MA, Klintworth GK. Immunohistochemical evidence of heterogeneity in macular corneal dystrophy. Am J Ophthalmol 1988;106:65-71.

10. Klintworth GK, Meyer R, Dennis R, et al. Macular corneal dystrophy: lack of keratan sulphate in the serum and cornea. Ophthalmic Paediatr Genet 1986;7:139-43.

11. Thonar EJ-MA, Meyer RF, Dennis RF, et al. Absence of normal keratan sulfate in the blood of patients with macular corneal dystrophy. Am J Ophthalmol 1986;102:561-9.

12. Meek KM, Quantock AJ, Elliott GF, et al. Macular corneal dystrophy: the macromolecular structure of the stroma observed using electron microscopy and synchrotron X-ray diffraction. Exp Eye Res 1989;49:941-58.

13. Quantock AJ, Meek KM, Thonar EJ-MA. Analysis of the high-angle synchrotron X-ray diffraction patterns obtained from macular dystrophy corneas. Cornea 1992;11:185-90.

14. Scott JE, Orford CR, Hughes EW. Proteoglycan-collagen arrangements in developing rat tail tendon. Biochem J 1981; 195:573-81.
15. Quantock AJ, Meek KM, Fullwood NJ, Zabel RW. Scheie's syndrome: the architecture of corneal collagen and distribution of corneal proteoglycans. Can J Ophthalmol 1993; 28:266-72.

16. Edward DP, Thonar EJ-MA, Srinivasan M, Yue BJYT, Tso MOM. Macular dystrophy of the cornea: a systemic disorder of keratan sulfate metabolism. Ophthalmology 1990;97: 1194-200.

17. Quantock AJ, Meek KM, Brittain P, Ridgway AEA, Thonar EJ-MA. Alteration of the stromal architecture and depletion of keratan sulphate proteoglycans in oedematous human corneas: histological, immunochemical and X-ray diffraction evidence. Tissue Cell 1991;23:595-606.

18. Spencer WH. In: Spencer WH, editor. Ophthalmic pathology: an atlas and textbook. Vol I, 3rd ed. Philadelphia: WB Saunders, 1985:327.

19. Benedek GB. Theory of transparency of the eye. Appl Optics 1971;10:459-73.

20. Hahn RA, Birk DE. $\beta$-D-xyloside alters dermatan sulfate proteoglycan synthesis and the organisation of the developing avian corneal stroma. Dev Biol 1992;115:383-93.

21. Scott JE. Morphometry of Cupromeronic blue-stained proteoglycan molecules in animal corneas, versus that of purified proteoglycans stained in vitro, implies that tertiary structures contribute to corneal ultrastructure. J Anat 1992; 180:155-64.

22. Scott JE. Proteoglycan-fibrillar collagen interactions. Biochem J 1988;252:313-23.

23. Rada JA,Schrecengost PK, Hassell JR. Regulation of corneal collagen fibrillogenesis by corneal keratan sulphate proteoglycan core protein [abstract]. Invest Ophthalmol Vis Sci 1991;32:1010.

24. Adachi E, Hayashi T. In vitro formation of hybrid fibrils of type $\mathrm{V}$ collagen and type I collagen: limited growth of type I collagen into thick fibrils by type V collagen. Connect Tissue Res 1986;14:257-66.

25. Birk DE, Fitch JM, Babiarz JP, Doane KJ, Linsenmayer TF. Collagen fibrillogenesis in vitro: interaction of types I and $\mathrm{V}$ regulates fibril diameter. J Cell Sci 1990;95:649-57. 